\title{
Erratum: Green's function method to study thin diffraction gratings [Phys. Rev. B 96, 205140 (2017)]
}

\author{
Daniel A. Travo, Rodrigo A. Muniz, M. Liscidini, and J. E. Sipe
}

(Received 27 October 2018; published 14 November 2018)

DOI: 10.1103/PhysRevB.98.199902

There were typographical errors in our published paper. Although these do not affect any of the results presented, we address these issues here.

The expression provided for $\bar{g}_{m m}$ just after Eq. (35) was written with $\kappa_{m}$ instead of $\kappa_{m}^{2}$ in several of its terms. The correct expression is

$$
\bar{g}_{m m}=\frac{i \tilde{\omega}^{2} D}{2 \epsilon_{0} w_{1}\left(\kappa_{m}\right)}\left[\begin{array}{ccc}
1-\frac{\kappa_{m}^{2}}{\tilde{\omega}^{2} \varepsilon_{1}} \sin ^{2} \phi & \frac{\kappa_{m}^{2}}{2 \tilde{\omega}^{2} \varepsilon_{1}} \sin 2 \phi & 0 \\
\frac{\kappa_{m}^{2}}{2 \tilde{\omega}^{2} \varepsilon_{1}} \sin 2 \phi & 1-\frac{\kappa_{m}^{2}}{\tilde{\omega}^{2} \varepsilon_{1}} \cos ^{2} \phi & 0 \\
0 & 0 & \frac{\kappa_{m}^{2}}{\tilde{\omega}^{2} \varepsilon_{1}}
\end{array}\right] .
$$

Additionally, in Fig. 3 the rays representing the wave vectors of the various diffracted components of the electric field within a given medium were drawn with different norms. Although the in-plane and perpendicular components of the wave vectors will differ for each diffracted component, their magnitude, and hence length in Fig. 3, should be the same. This is corrected in Fig. 3.

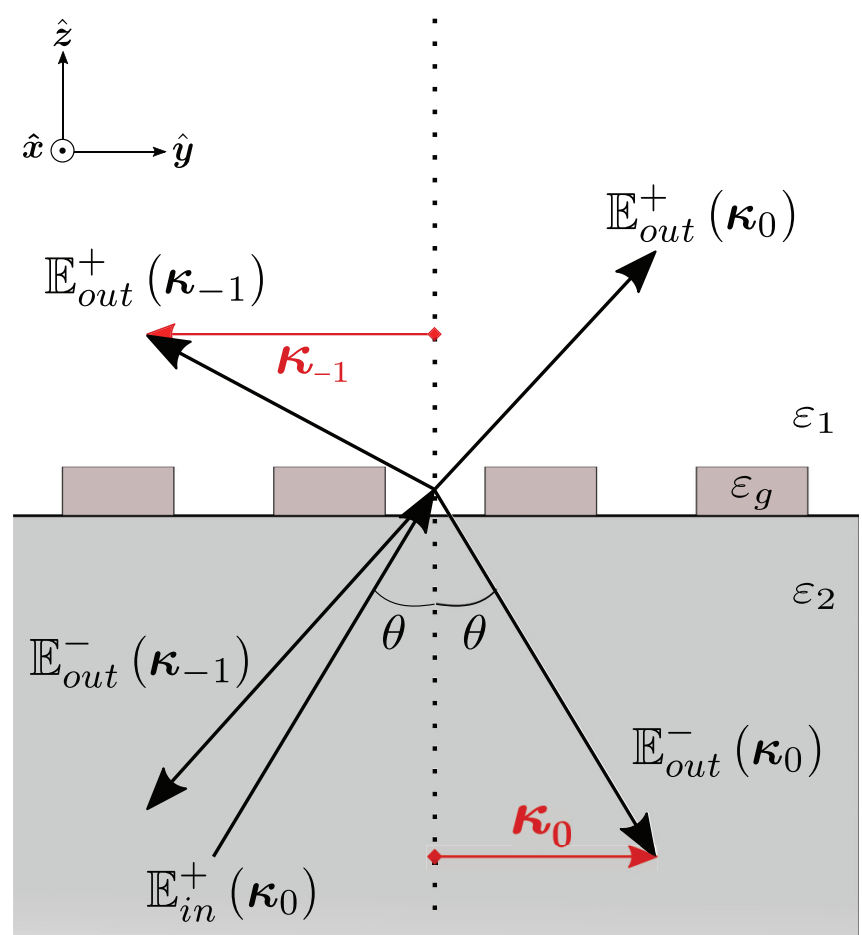

FIG. 3. A simple one-dimensional grating configuration with the grating oriented such that $\hat{\boldsymbol{e}}=\hat{\boldsymbol{y}}$. Incident, reflected, transmitted, and diffracted rays are shown by black (thick) lines and labeled by the notation used to indicate their field amplitudes; the projections of the wave vectors on the $x y$ plane are shown by red (thin) lines. 
Finally, the expressions for the $p$-polarized transmission and reflection matrices, given in Eq. (53), were missing a factor of $\bar{w}_{1}^{-1}$ in each of their first terms. The corrected expressions are given in Eq. (53). Again, this does not affect any of the published results,

$$
\begin{aligned}
\mathbb{T}_{g, p} & =\left[\overline{1}_{1}-\frac{i D}{2 \varepsilon_{1}} \bar{w}_{1}^{-1} \bar{\kappa} \bar{\chi}^{\perp} \bar{\kappa}\right]^{-1}+\bar{\beta}\left[\overline{1}_{1}-\frac{i D}{2 \varepsilon_{1}} \bar{\chi}^{\|} \bar{w}_{1}\right]^{-1} \bar{\beta}-\overline{1}_{1}, \\
\mathbb{R}_{g, p} & =\left[\overline{1}_{1}-\frac{i D}{2 \varepsilon_{1}} \bar{w}_{1}^{-1} \bar{\kappa} \bar{\chi}^{\perp} \bar{\kappa}\right]^{-1}-\bar{\beta}\left[\overline{1}_{1}-\frac{i D}{2 \varepsilon_{1}} \bar{\chi}^{\|} \bar{w}_{1}\right]^{-1} \bar{\beta} .
\end{aligned}
$$

